\title{
Characteristic polynomials of skew-adjacency matrices of oriented graphs
}

\author{
Yaoping Hou \\ Department of Mathematics \\ Hunan Normal University \\ Changsha, Hunan 410081, China \\ yphou@hunnu.edu.cn
}

\author{
Tiangang Lei \\ Department of Mathematical and Physical Sciences \\ National Nature Science Foundation of China \\ Beijing 100875, China \\ leitg@mail.nsfc.gov.cn
}

Submitted: Jan 18, 2011; Accepted: Jul 4, 2011; Published: Aug 5, 2011

Mathematics Subject Classification: 05C20, 05C50

\begin{abstract}
An oriented graph $G^{\sigma}$ is a simple undirected graph $G$ with an orientation, which assigns to each edge a direction so that $G^{\sigma}$ becomes a directed graph. $G$ is called the underlying graph of $G^{\sigma}$ and we denote by $S\left(G^{\sigma}\right)$ the skew-adjacency matrix of $G^{\sigma}$ and its spectrum $\operatorname{Sp}\left(G^{\sigma}\right)$ is called the skew-spectrum of $G^{\sigma}$. In this paper, the coefficients of the characteristic polynomial of the skew-adjacency matrix $S\left(G^{\sigma}\right)$ are given in terms of $G^{\sigma}$ and as its applications, new combinatorial proofs of known results are obtained and new families of oriented bipartite graphs $G^{\sigma}$ with $S p\left(G^{\sigma}\right)=$ i $S p(G)$ are given.
\end{abstract}

\section{Introduction}

All undirected graphs in this paper are simple and finite. Let $G$ be a graph with $n$ vertices and $A(G)=\left(a_{i, j}\right)$ the adjacency matrix of $G$, where $a_{i, j}=a_{j, i}=1$ if there is an edge $i j$ between vertices $i$ and $j$ in $G$ (denoted by $i \sim j$ ), otherwise $a_{i, j}=a_{j, i}=0$. We call $G$ nonsingular if the matrix $A(G)$ is nonsingular. The characteristic polynomial $P(G ; x)=\operatorname{det}(x I-A(G))$ of $A(G)$, where $I$ stands for the identity matrix of order $n$, is said to be the characteristic polynomial of the graph $G$. The $n$ roots of the polynomial $P(G ; x)$ are said to be the eigenvalues of the graph $G$. Since $A(G)$ is symmetric, all eigenvalues of $A(G)$ are real and we denote by $S p(G)$ the adjacency spectrum of $G$.

Let $G^{\sigma}$ (or $\vec{G}$ ) be a graph with an orientation, which assigns to each edge of $G$ a direction so that $G^{\sigma}$ becomes a directed graph. The skew-adjacency matrix $S\left(G^{\sigma}\right)=\left(s_{i, j}\right)$ is real skew symmetric matrix, where $s_{i, j}=1$ and $s_{j, i}=-1$ if $i \rightarrow j$ is an arc of $G^{\sigma}$, otherwise $s_{i, j}=s_{j, i}=0$. The skew-spectrum $S p\left(G^{\sigma}\right)$ of $G^{\sigma}$ is defined as the spectrum of $S\left(G^{\sigma}\right)$. Note that $S p\left(G^{\sigma}\right)$ consists of only purely imaginary eigenvalues because $S\left(G^{\sigma}\right)$ is real skew symmetric. 
Unlike the adjacency matrix of a graph, there is little research on the skew-adjacency matrix $S\left(G^{\sigma}\right)$, except that in enumeration of perfect matchings of a graph, see [9] and references therein, where the square of the number of perfect matchings of a graph $G$ with a Pfaffian orientation is the determinant of the skew-adjacency matrix $S\left(G^{\sigma}\right)$.

Recently, the skew-energy of $G^{\sigma}$ was defined as the energy of matrix $S\left(G^{\sigma}\right)$, that is,

$$
\mathcal{E}\left(G^{\sigma}\right)=\sum_{\lambda \in S p\left(G^{\sigma}\right)}|\lambda|
$$

The concept of the energy of an undirected graph was introduced by Gutman and there has been a constant streams of papers devoted to this topic. The concept of the skewenergy of a simple directed graph (that is, oriented graph) was introduced by Adiga, Balakrishnan and So, and some basic facts are discussed and some open problems are proposed [1], such as,

- Problem 1: Interpret all the coefficients of the characteristic polynomial of $S\left(G^{\sigma}\right)$.

- Problem 2: Find new families of oriented graphs $G^{\sigma}$ with $\mathcal{E}\left(G^{\sigma}\right)=\mathcal{E}(G)$.

The motivation of this paper is to address the above two open problems. In section 2 we derive the coefficients of the characteristic polynomial of $S\left(G^{\sigma}\right)$ in terms of $G^{\sigma}$, which is similar to the result of the coefficients of the characteristic polynomial of the adjacency matrix $A(G)$. In section 3 we give some applications of the coefficients theorem: the new combinatorial proofs of known results in [10] are obtained (that is, $S p\left(G^{\sigma}\right)=\mathbf{i} S p(G)$ for some orientation $\sigma$ if and only if $G$ is bipartite and $S p\left(G^{\sigma}\right)=\mathbf{i} S p(G)$ for any orientation $G^{\sigma}$ of $G$ if and only if $G$ is acyclic) and some new families of oriented bipartite graphs $G^{\sigma}$ with $\mathcal{E}\left(G^{\sigma}\right)=\mathcal{E}(G)$ are given.

\section{The skew-characteristic polynomial of $G^{\sigma}$}

Let $G$ be a graph. A linear subgraph $L$ of $G$ is a disjoint union of some edges and some cycles in $G$. A $k$-matching $\mathcal{M}$ in $G$ is a disjoint union of $k$-edges. If $2 k$ is the order of $G$, then a $k$ - matching of $G$ is called a perfect matching of $G$.

Let $G$ be a graph and $A(G)$ be its adjacency matrix and characteristic polynomial of $G$ be

$$
P(G ; x)=\operatorname{det}(x I-A)=\sum_{i=0}^{n} a_{i} x^{n-i} .
$$

Then $a_{0}(G)=1, a_{1}(G)=0$, and $-a_{2}(G)$ is the number of edges in $G$. In general, we have (see [7])

$$
a_{i}=\sum_{L \in \mathcal{L}_{i}}(-1)^{p_{1}(L)}(-2)^{p_{2}(L)}
$$

where $\mathcal{L}_{i}$ denotes the set of all linear subgraphs $L$ of $G$ with $i$ vertices, $p_{1}(L)$ is the number of components of size 2 in $L$ and $p_{2}(L)$ is the number of cycles in $L$. If $G$ is bipartite, then $a_{i}=0$ for all odd $i$, and 


$$
P(G ; x)=\sum_{i=0}^{\left\lfloor\frac{n}{2}\right\rfloor}(-1)^{i} b_{2 i}(G) x^{n-2 i},
$$

where all $b_{2 i}=(-1)^{i} a_{2 i}$ are nonnegative [4, p. 147].

Let $G$ be a graph and $G^{\sigma}$ be an orientation of $G$ and $S\left(G^{\sigma}\right)$ be the skew-adjacency matrix of $G^{\sigma}$. Denote the characteristic polynomial of $S\left(G^{\sigma}\right)$ by

$$
P\left(G^{\sigma} ; x\right)=\operatorname{det}(x I-S)=\sum_{i=0}^{n} c_{i} x^{n-i}
$$

Then (i) $c_{0}=1$, (ii) $c_{2}$ is the number of edges of $G$, (iii) $c_{i} \geq 0$ for all $i$ and (iv) all $c_{i}=0$ for all odd $i$ since the determinant of any skew symmetric matrix is nonnegative and is 0 if its order is odd. In this section we give $c_{i}$ in term of $G^{\sigma}$ in general. It is based on the combinatorial definition of the determinant of a matrix [6, Section 9.1].

Recall the definition of the determinant of a matrix $M=\left(m_{i, j}\right)$ is

$$
\operatorname{det} M=\sum_{\tau \in \operatorname{Sym}(n)} \operatorname{sign}(\tau) m_{1, \tau(1)} m_{2, \tau(2)} \cdots m_{n, \tau(n)},
$$

where the summation extends over the set $\operatorname{Sym}(n)$ of all permutations $\tau$ of $\{1,2, \ldots, n\}$. Suppose that the permutation $\tau$ consists of $k$ permutation cycles of sizes $\ell_{1}, \ell_{2}, \ldots, \ell_{k}$, respectively, where $\ell_{1}+\ell_{2}+\cdots+\ell_{k}=n$. Then $\operatorname{sign}(\tau)$ can be computed by

$$
\operatorname{sign}(\tau)=(-1)^{\ell_{1}-1+\ell_{2}-1+\cdots+\ell_{k}-1}=(-1)^{n}(-1)^{k} .
$$

Let $D_{n}$ be the complete digraph with vertex set $\{1,2, \ldots, n\}$ in which each ordered pair $(i, j)$ of vertices forms an arc of $D_{n}$. We assign to each $\operatorname{arc}(i, j)$ of $D_{n}$ the weight $m_{i, j}$ and thereby obtain a weighted digraph. The weight of a directed cycle $\gamma: i_{1} \rightarrow i_{2} \rightarrow \cdots \rightarrow$ $i_{t} \rightarrow i_{1}$ is defined to be

$$
-m_{i_{1}, i_{2}} \cdots m_{i_{t-1}, i_{t}} m_{i_{t}, i_{1}},
$$

the negative of all the product of the weights of arcs.

Let $\tau$ be a permutation of $\{1,2, \ldots, n\}$ as above. The permutation digraph $D(\tau)$ is the digraph with vertices $\{1,2, \ldots, n\}$ and with the $n$ arcs $\{(i, \tau(i)): i=1,2, \ldots, n\}$. The digraph $D(\tau)$ is a spanning sub-digraph of the complete digraph $D_{n}$. The directed cycles of $D(\tau)$ are in one-to-one correspondence with the permutation cycles of $\tau$ and the arc sets of these directed cycles partition the set of arcs of $D(\tau)$. The weight wt $(D \tau)$ of the permutation digraph $D(\tau)$ is defined to be the product of the weights of its direct cycles, $w t(D(\tau))=(-1)^{k} m_{1, \tau(1)} m_{2, \tau(2)} m_{n, \tau(n)}$. Using (2.5) and (2.6), we obtain

$$
\operatorname{det}(M)=(-1)^{n} \sum_{\tau \in \operatorname{Sym}(n)} w t(D(\tau))
$$

Let $\mathcal{E}(n)$ denote the set of all permutations $\tau$ of $\{1,2, \ldots, n\}$ such that the size of all permutation cycles of $\tau$ are even. 
Lemma 2.1 [8, Lemma 2.1] If $M=\left(m_{i, j}\right)$ is an $n \times n$ skew symmetric matrix then

$$
\operatorname{det} M=\sum_{\tau \in \mathcal{E}(n)} \operatorname{sign}(\tau) m_{1, \tau(1)} \cdots m_{n, \tau(n)} .
$$

If $M=\left(m_{i, j}\right)$ is an $n \times n$ skew symmetric matrix then

$$
\operatorname{det}(M)=(-1)^{n} \sum_{\tau \in \mathcal{E}(n)} w t(D(\tau)) .
$$

We need also following concepts from [9] in order to interpret all coefficients $c_{2 i}$ in term of $G^{\sigma}$.

Let $C$ be an undirected even cycle of $G^{\sigma}$. Now regardless of which of the possible routing around $C$ is chosen, if $C$ contains an even number of oriented edge whose orientation agrees with the routing, then $C$ also contains an even number of edges whose orientation is opposite to the routing. Hence the following definition is independent of the routing chosen.

If $C$ be any undirected even cycle of $G^{\sigma}$, we say $C$ is evenly oriented relative to $G^{\sigma}$ if it has an even number of edges oriented in the direction of the routing. Otherwise $C$ is oddly oriented.

Let $S=\left(s_{i j}\right)$ be skew-adjacency matrix of an oriented graph $G^{\sigma}$. Note that each undirected cycle $C$ of $G^{\sigma}$ correspondences two permutation cycles, and the weights of these two permutation digraphs are -1 if $C$ is evenly oriented relative to $G^{\sigma}$ and +1 if $C$ is oddly oriented.

We call a linear subgraph $L$ of $G$ evenly linear if $L$ contains no cycle with odd length and denote by $\mathcal{E} \mathcal{L}_{i}(G)$ ( or $\mathcal{E} \mathcal{L}_{i}$ for short) the set of all evenly linear subgraphs of $G$ with $i$ vertices. For a linear subgraph $L \in \mathcal{E} \mathcal{L}_{i}$ denote by $p_{e}(L)$ (resp., $\left.p_{o}(L)\right)$ the number of evenly (resp., oddly) oriented cycles in $L$ relative to $G^{\sigma}$. For a linear subgraph $L \in \mathcal{E} \mathcal{L}_{n}$, $L$ contributes $(-2)^{p_{e}(L)} 2^{p_{o}(L)}$ to the determinant of $S\left(G^{\sigma}\right)$.

Summarizing the above we have

Lemma 2.2 If $S\left(G^{\sigma}\right)=\left(s_{i, j}\right)$ is an $n \times n$ skew-adjacency matrix of the orientation $G^{\sigma}$ of a graph $G$. Then

$$
\operatorname{det} S\left(G^{\sigma}\right)=\sum_{L \in \mathcal{E} \mathcal{L}_{n}}(-2)^{p_{e}(L)} 2^{p_{o}(L)}
$$

where $p_{e}(L)$ is the number of evenly oriented cycles of $L$ relative to $G^{\sigma}$ and $p_{o}(S)$ is the number of oddly oriented cycles of $L$ relative to $G^{\sigma}$, respectively.

Note that if $n$ is odd then $\mathcal{E} \mathcal{L}_{n}$ is empty and hence $\operatorname{det} S\left(G^{\sigma}\right)=0$.

As $(-1)^{i} c_{i}$ is the summation of determinants of all principal $i \times i$ submatrices $S\left(G^{\sigma}\right)$, using Lemma 2.2, we have

Theorem 2.3 Let $G$ be a graph and $G^{\sigma}$ be an orientation of $G$. Then

$$
c_{i}=\sum_{L \in \mathcal{E} \mathcal{L}_{i}}(-2)^{p_{e}(L)} 2^{p_{o}(L)},
$$


where $p_{e}(L)$ is the number of evenly oriented cycles of $L$ relative to $G^{\sigma}$ and $p_{o}(S)$ is the number of oddly oriented cycles of $L$ relative to $G^{\sigma}$, respectively. In particular, $c_{i}=0$ if $i$ is odd.

As applications of the above theorem, we can obtain the following result which can be used to find recursions for the characteristic polynomial of some skew-adjacency matrices.

Theorem 2.4 Let $e=u v$ be an edge of $G$, then

$P\left(G^{\sigma} ; x\right)=P\left(G^{\sigma}-e ; x\right)+P\left(G^{\sigma}-u-v ; x\right)+2 \sum_{e \in C \in \operatorname{Od}\left(G^{\sigma}\right)} P\left(G^{\sigma}-C ; x\right)-2 \sum_{e \in C \in E v\left(G^{\sigma}\right)} P\left(G^{\sigma}-C ; x\right)$.

Proof. Every evenly linear subgraph $L$ of $G$ with $i$ vertices must belong to one of the following four kinds:

(1). $\mathcal{E}_{1}: L$ does not contain the edge $e$;

(2). $\mathcal{E}_{2}: L$ contains the edge $e$ but $e$ is not in any cycle component of $L$;

(3). $\mathcal{E}_{3}: L$ contains the edge $e$ and $e$ is contained in some oddly oriented cycle component $C$ of $L$;

(4). $\mathcal{E}_{4}: L$ contains the edge $e$ and $e$ is contained in some evenly oriented cycle component $C$ in $L$.

Note that any evenly linear subgraph $L$ with $i$ vertices which does not use $e$ is an evenly linear subgraph with $i$ vertices of $G-e$. If an evenly linear subgraph $L$ belongs $\mathcal{E}_{2}$, then the edge $e$ is a component and $L$ determines an evenly linear subgraph $L^{\prime}$ of $G-u-v$ with $i-2$ vertices such that $L=e \cup L^{\prime}$. For any evenly linear subgraph $L$ belongs to $\mathcal{E}_{3}$ $\left(\right.$ or $\left.\mathcal{E}_{4}\right), L$ determines an evenly linear subgraph $L^{\prime}$ of $G-C$ with $i-|C|$ vertices for some oddly (resp., evenly) oriented cycle $C$ in $G^{\sigma}$ such that $L=C \cup L^{\prime}$. Hence,

$$
\begin{aligned}
& c_{i}\left(G^{\sigma}\right)=\sum_{L \in \mathcal{E} \mathcal{L}_{i}(G)}(-2)^{p_{e}(L)} 2^{p_{o}(L)} \\
& =\sum_{L \in \mathcal{E}_{1}}(-2)^{p_{e}(L)} 2^{p_{o}(L)}+\sum_{L \in \mathcal{E}_{2}}(-2)^{p_{e}(L)} 2^{p_{o}(L)} \\
& +\sum_{L \in \mathcal{E}_{3}}(-2)^{p_{e}(L)} 2^{p_{o}(L)}+\sum_{L \in \mathcal{E}_{4}}(-2)^{p_{e}(L)} 2^{p_{o}(L)} \\
& =\sum_{L^{\prime} \in \mathcal{E} \mathcal{L}_{i}(G-e)}(-2)^{p_{e}\left(L^{\prime}\right)} 2^{p_{o}\left(L^{\prime}\right)}+\sum_{L^{\prime} \in \mathcal{E} \mathcal{L}_{i-2}(G-u-v)}(-2)^{p_{e}\left(L^{\prime}\right)} 2^{p_{o}\left(L^{\prime}\right)} \\
& +2 \sum_{e \in C \in \operatorname{Od}\left(G^{\sigma}\right)} \sum_{L^{\prime} \in \mathcal{E} \mathcal{L}_{i-|C|}(G-C)}(-2)^{p_{e}\left(L^{\prime}\right)} 2^{p_{o}\left(L^{\prime}\right)} \\
& -2 \sum_{e \in C \in E v\left(G^{\sigma}\right)} \sum_{L^{\prime} \in \mathcal{E} \mathcal{L}_{i-|C|}(G-C)}(-2)^{p_{e}\left(L^{\prime}\right)} 2^{p_{o}\left(L^{\prime}\right)} \\
& =c_{i}\left(G^{\sigma}-e\right)+c_{i-2}\left(G^{\sigma}-u-v\right)+2 \sum_{e \in C \in \operatorname{Od}\left(G^{\sigma}\right)} c_{i-|C|}\left(G^{\sigma}-C\right) \\
& -2 \sum_{e \in C \in E v\left(G^{\sigma}\right)} c_{i-|C|}\left(G^{\sigma}-C\right),
\end{aligned}
$$


where $\operatorname{Od}\left(G^{\sigma}\right)$ (resp., $E v\left(G^{\sigma}\right)$ ) is the set of all oddly (resp., evenly) oriented (even) cycles of $G^{\sigma}$. Therefore, the result follows.

Corollary 2.5 Let $e=u v$ be an edge of $G$ that is on no even cycle in $G$. Then

$$
P\left(G^{\sigma} ; x\right)=P\left(G^{\sigma}-e ; x\right)+P\left(G^{\sigma}-u-v ; x\right) .
$$

Example 2.6 Let $S_{n, 3}$ be the unicyclic graph obtained from the star of $n$ vertices by adding an edge and $S_{n, 3}^{\sigma}$ be any orientation of $S_{n, 3}$. Then by (2.9),

$$
P\left(S_{n, 3}^{\sigma} ; x\right)=x^{n}+n x^{n-2}+(n-3) x^{n-4} .
$$

Let $S_{n, 4}$ be the unicycle graph obtained from the cycle $C_{4}$ by adding $n-4$ pendent vertices to a vertex of $C_{4}$ and let $S_{n, 4}^{o}$ (resp., $S_{n, 4}^{e}$ ) be an orientation of graph $S_{n, 4}$ such that the unique cycle $C_{4}$ in $S_{n, 4}$ is oddly (resp., evenly) oriented relative to $S_{n, 4}^{o}$. Then

$$
\begin{aligned}
& P\left(S_{n, 4}^{o} ; x\right)=x^{n}+n x^{n-2}+(2 n-4) x^{n-4}, \\
& P\left(S_{n, 4}^{e} ; x\right)=x^{n}+n x^{n-2}+(2 n-8) x^{n-4} .
\end{aligned}
$$

Let $C_{n}$ and $P_{n}$ be the cycle graph and the path graph with $n$ vertices, respectively. In what follows we compute the characteristic polynomial of the skew-adjacency matrix of any orientation of $C_{n}$ and $P_{n}$. Letting $\mathbf{i}=\sqrt{-1}$ and $x=2 \mathbf{i} \sin \tau$, we have $P\left(P_{1}^{\sigma}, x\right)=$ $2 \mathbf{i} \sin \tau, P\left(P_{2}^{\sigma}, x\right)=2 \cos 2 \tau-1=\frac{\cos 3 \tau}{\cos \tau}$, and $P\left(P_{n}^{\sigma} ; x\right)=x P\left(P_{n-1}^{\sigma} ; x\right)+P\left(P_{n-2}^{\sigma} ; x\right)$ for $n \geq 3$. Using the identities $\sin (\theta+\varphi)-\sin (\theta-\varphi)=2 \cos \theta \sin \varphi$ and $\cos (\theta+\varphi)-\cos (\theta-\varphi)=$ $-2 \sin \theta \sin \varphi$ with $\theta=n \tau$ and $\varphi=\tau$, it follows that the solution of the recursion is

\section{Example 2.7}

$$
P\left(P_{n}{ }^{\sigma} ; x\right)= \begin{cases}\frac{\cos (n+1) \tau}{\cos \tau}, & n \text { is even } \\ \frac{\operatorname{isin}(n+1) \tau}{\cos \tau}, & n \text { is odd }\end{cases}
$$

When $\pi / 2<\tau<-\pi / 2$, then values of $x=2 \mathbf{i} \sin \tau$ are distinct and balanced. To obtain the skew-spectrum of $P_{n}^{\sigma}$, if $j=1,2, \ldots, n$, we may take $\tau=(n+1-2 j) \frac{\pi}{2(n+1)}$ when $n$ is even and also when $n$ is odd. Since $\sin \tau=\cos \left(\frac{\pi}{2}-\tau\right)$, the skew-spectrum of $P_{n}{ }^{\sigma}$ is $\left\{2 \mathbf{i} \cos \frac{j \pi}{n+1} \mid j=1,2, \ldots, n\right\}$.

Using Corollary 2.4, for any orientation $C_{n}^{\sigma}$ of the cycle $C_{n}$, we have $P\left(C_{n}{ }^{\sigma} ; x\right)= \begin{cases}P\left(P_{n}^{\sigma} ; x\right)+P\left(P_{n-2}^{\sigma} ; x\right)+2, & n \text { is even and the cycle is oddly oriented; } \\ P\left(P_{n}^{\sigma} ; x\right)+P\left(P_{n-2}^{\sigma} ; x\right)-2, & n \text { is even and the cycle is evenly oriented; } \\ P\left(P_{n}^{\sigma} ; x\right)+P\left(P_{n-2}^{\sigma} ; x\right), & n \text { is odd. }\end{cases}$

Hence, by the Example 2.7, we have 


\section{Example 2.8}

$$
P\left(C_{n}{ }^{\sigma} ; x\right)= \begin{cases}2 \cos n \tau+2, & n \text { is even and the cycle is oddly oriented; } \\ 2 \cos n \tau-2, & n \text { is even and the cycle is evenly oriented; } \\ 2 \mathbf{i} \sin n \tau, & n \text { is odd. }\end{cases}
$$

Hence the skew-spectrum of $C_{n}{ }^{\sigma}$ is $\left\{2 \mathbf{i} \sin \frac{2 j \pi}{n} \mid j=1,2, \ldots, n\right\}$ if $n$ is odd, and $\left\{2 \mathbf{i} \sin \frac{(2 j-1) \pi}{n} \mid j=1,2, \ldots, n\right\}$ if $n$ is even and the cycle is oddly oriented, and $\left\{2 \mathbf{i} \sin \frac{2 j \pi}{n} \mid j=1,2, \ldots, n\right\}$ if $n$ is even and the cycle is evenly oriented.

\section{Oriented graphs $G^{\sigma}$ with $\operatorname{Sp}\left(G^{\sigma}\right)=\mathrm{i} \operatorname{Sp}(G)$}

Let $G$ be a graph and $G^{\sigma}$ be an orientation of $G$. The characteristic polynomials of $G$ and $G^{\sigma}$ are expressed as (2.1) and (2.4), respectively. Because the roots of $P\left(G^{\sigma} ; x\right)$ are pure imaginary and occur in complex conjugate pairs, while the roots of $P(G ; x)$ are all real, it follows that $S p\left(G^{\sigma}\right)=\mathbf{i} S p(G)$ if and only if $P(G ; x)=\sum_{i=0}^{n} a_{i} x^{n-i}=$ $x^{n-2 r} \prod_{i=1}^{r}\left(x^{2}-\lambda_{i}^{2}\right)$ and $P(G ; x)=\sum_{i=0}^{n} c_{i} x^{n-i}=x^{n-2 r} \prod_{i=1}^{r}\left(x^{2}+\lambda_{i}^{2}\right)$ for some non-zero real numbers $\lambda_{1}, \lambda_{2}, \ldots, \lambda_{r}$ if and only if

$$
a_{2 i}=(-1)^{i} c_{2 i}, a_{2 i+1}=c_{2 i+1}=0,
$$

where $i=0,1, \ldots,\left\lfloor\frac{n}{2}\right\rfloor$.

Let $G^{\sigma}$ be an orientation of a graph $G$. An even cycle $C_{2 \ell}$ is said to be oriented uniformly if $C_{2 \ell}$ is oddly (resp., evenly) oriented relative to $G^{\sigma}$ when $\ell$ is odd (resp., even).

Lemma 3.1 Let $G$ be a bipartite graph and $G^{\sigma}$ be an orientation of $G$. If every even cycle is oriented uniformly then $\operatorname{Sp}\left(G^{\sigma}\right)=\mathbf{i} S p(G)$.

Proof. Since $G$ is bipartite, all cycles in $G$ are even and all linear subgraphs are even. Then $a_{2 i+1}=0$ for all $i$. Since every even cycle is oriented uniformly, for every cycle $C_{2 \ell}$ with length $2 \ell, C_{2 \ell}$ is evenly oriented relative to $G^{\sigma}$ if and only if $\ell$ is even. Thus $(-1)^{p_{e}\left(C_{2 \ell}\right)}=(-1)^{\ell+1}$.

By Eqs (2.2) and (2.9), we have

$$
\begin{aligned}
(-1)^{i} a_{2 i} & =m(G, i)+\sum_{L \in \mathcal{C} \mathcal{L}_{2 i}}(-1)^{p_{1}(L)+i}(-2)^{p_{2}(L)}, \\
c_{2 i} & =m(G, i)+\sum_{L \in \mathcal{C} \mathcal{L}_{2 i}}(-2)^{p_{e}(L)} 2^{p_{o}(L)}
\end{aligned}
$$

where $m(G, i)$ is the number of matchings with $i$ edges and $\mathcal{C} \mathcal{L}_{2 i}$ is the set of all linear subgraphs with $2 i$ vertices of $G$ and with at least one cycle.

For a linear subgraph $L \in \mathcal{C} \mathcal{L}_{2 i}$ of $G$, assume that $L$ contains the cycles $C_{2 \ell_{1}}, \ldots, C_{2 \ell_{2}}$. Then the number of components of $L$ that are single edge is $p_{1}(L)=i-\sum_{j=1}^{p_{2}(L)} \ell_{j}$. Hence 

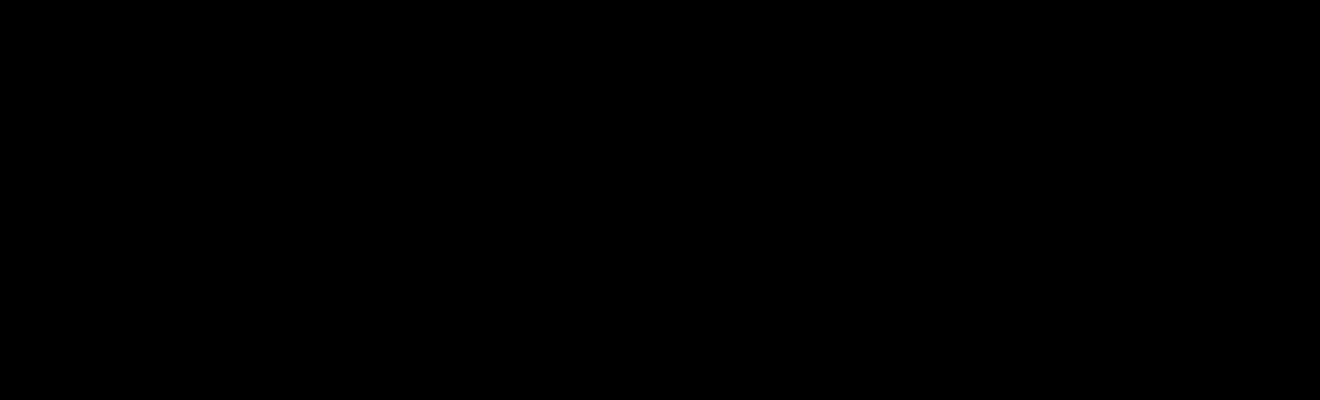

Figure 1: A graph $G$ and an orientation $G^{\sigma}$ with all even cycles oriented uniformly.

$(-1)^{p_{1}(L)+i}=(-1)^{\sum_{j=1}^{p_{2}(L)} \ell_{j}}$. Therefore $L$ contributes $(-1)^{\ell_{1}+1} \cdots(-1)^{\ell_{p_{2}}+1}(-2)^{p_{2}(L)}=$ $(-1)^{p_{1}(L)+i}(-2)^{p_{2}(L)}$ in $c_{2 i}$. Thus $(-1)^{i} a_{2 i}=c_{2 i}$ by Eqs. (3.2) and (3.3) and the proof is completed.

The following corollary provides a new family of oriented bipartite graphs $G^{\sigma}$ with $S p\left(G^{\sigma}\right)=\mathbf{i} S p(G)$ and hence $\mathcal{E}\left(G^{\sigma}\right)=\mathcal{E}(G)$.

Corollary 3.2 Let $G$ be a graph whose blocks are $K_{2}$ or even cycles. If all even cycles of $G$ are oriented uniformly in $G^{\sigma}$ then $S p\left(G^{\sigma}\right)=\mathbf{i} S p(G)$ and hence $\mathcal{E}\left(G^{\sigma}\right)=\mathcal{E}(G)$.

The following two results appeared in [10]. The proofs there are based on matrix theory. Now we give proofs that are more combinatorial.

Theorem 3.3 A graph $G$ is bipartite if and only if there is an orientation $\sigma$ such that $S p\left(G^{\sigma}\right)=\mathbf{i} S p(G)$.

Proof. (Sufficiency) If there is an orientation $\sigma$ such that $S p\left(G^{\sigma}\right)=\mathbf{i} S p(G)$ then $a_{2 i+1}=$ $c_{2 i+1}=0$. Hence $G$ is bipartite.

(Necessity) If $G$ is a bipartite graph with vertices partition $V=V_{1} \cup V_{2}$. Let $G^{\sigma}$ be the orientation such that all arcs are from $V_{1}$ to $V_{2}$. Then $a_{2 i+1}=0$ for all $i$, and every even cycle is oriented uniformly relative to $G^{\sigma}$. Thus $S p\left(G^{\sigma}\right)=\mathbf{i} S p(G)$ by Lemma 3.1

We call a graph $G$ acyclic (or a forest) if $G$ contains no cycles. A tree is a connected and acyclic graph.

Theorem 3.4 Let $G$ be a graph. Then $\mathbf{i} S p(G)=S p\left(G^{\sigma}\right)$ for any orientation $G^{\sigma}$ if and only if $G$ is acyclic.

Proof. (Sufficiency) If $G$ is acyclic, then $a_{2 i+1}=0$ and $a_{2 i}=(-1)^{i} m(G, i)$ and $c_{2 i}=$ $m(G, i)$ and hence $\mathbf{i} S p(G)=S p\left(G^{\sigma}\right)$ by the first paragraph of this section.

(Necessity) Suppose that $G$ is not acyclic, then $G$ contains at least a cycle. $G$ is bipartite by $\mathrm{i} S p(G)=S p_{S}\left(G^{\sigma}\right)$ and Theorem 3.3. Let the length of shortest cycle of $G$ be $g$, then $g$ is even, say $g=2 r$. Then $(-1)^{r} a_{g}=m(G, r)+(-1)^{r+1} 2 n\left(G, C_{g}\right)$ and 
$c_{g}=m(G, r)+2 n_{o}\left(G^{\sigma}, C_{g}\right)-2 n_{e}\left(G^{\sigma}, C_{g}\right)$, where $n_{o}\left(G^{\sigma}, C_{g}\right)\left(n_{e}\left(G^{\sigma}, C_{g}\right)\right)$ is the number of oddly (resp., evenly) oriented cycles in $G$ of length $g$ relative to $G^{\sigma}$ and $n\left(G, C_{g}\right)$ is the number of cycles in $G$ of length $g$. Note that $n_{o}\left(G^{\sigma}, C_{g}\right)+n_{e}\left(G^{\sigma}, C_{g}\right)=n\left(G, C_{g}\right)$. As in the proof of Theorem 3.3, let $G$ have the orientation $G^{\sigma}$ where all edges are directed from $V_{1}$ to $V_{2}$. For this orientation, $2 n_{o}\left(G^{\sigma}, C_{g}\right)-2 n_{e}\left(G^{\sigma}, C_{g}\right)$ equals $2 n_{o}\left(G^{\sigma}, C_{g}\right)$ if $r$ odd and $-2 n_{e}\left(G^{\sigma}, C_{g}\right)$ if $r$ is even. Thus reversing the direction of an edge that is on at least one cycle of length $g$ must change $2 n_{o}\left(G^{\sigma}, C_{g}\right)-2 n_{e}\left(G^{\sigma}, C_{g}\right)$ and so must change $c_{g}$. Hence $(-1)^{r+1} 2 n\left(G, C_{g}\right) \neq 2 n_{o}\left(G^{\sigma}, C_{g}\right)-2 n_{e}\left(G^{\sigma}, C_{g}\right)$. That is, $(-1)^{r} a_{2 r} \neq c_{2 r}$, which is contradiction with $\mathrm{i} S p(G) \neq S p\left(G^{\sigma}\right)$.

From the above Theorem 3.4, if $T$ is a tree and $\vec{T}$ is any orientation of $T$ then $S p(\vec{T})=\mathbf{i} S p(T)$. In what follows we provide another interesting family of oriented graphs $G^{\sigma}$ with $S p\left(G^{\sigma}\right)=\mathbf{i} S p(G)$ and hence with $\mathcal{E}\left(G^{\sigma}\right)=\mathcal{E}(G)$

Let $T$ be a tree with a perfect matching $\mathcal{M}$ (in this case, $T$ has a unique perfect matching) and $\vec{T}$ be an orientation of $T$. Note that the adjacency matrix $A(T)$ of $T$ and skew-adjacency matrix $S(\vec{T})$ of $\vec{T}$ are nonsingular if and only if $T$ has a perfect matching. In order to describe the inverses of $A(T)$ and $S(T)$, the following definition of an alternating path is taken from Buckley, Doty and Harary [5, p.156].

Definition 3.5 Let $G$ be a graph with a perfect matching $\mathcal{M}$. A path in $G: P(i, j)=$ $i_{1} i_{2} \cdots i_{2 k}$ (where $i_{1}=i, i_{2 k}=j$ ) from a vertex $i$ to a vertex $j$ is said to be an alternating path if the edges $i_{1} i_{2}, i_{3} i_{4}, \cdots, i_{2 k-1} i_{2 k}$ are edges in the perfect matching $\mathcal{M}$.

For a tree with a perfect matching, there is at most one alternating path between any pair of vertices. Note that if $P(i, j)$ is an alternating path between vertices $i$ and $j$, then the number of edges in $P(i, j)$ which are not in $\mathcal{M}$ is $\frac{|P(i, j)|-1}{2}$, where $|P(i, j)|$ is the number of the edges in the path $P(i, j)$.

Proposition 3.6 (Buckley, Doty and Harary [5, Theorem 3]) Let $T$ be a nonsingular tree on $n$ vertices and $A$ be its adjacency matrix. Let $B=\left(b_{i, j}\right)$, where

$$
b_{i, j}= \begin{cases}(-1)^{\frac{|P(i, j)|-1}{2}}, & \text { if there is an alternating path } P(i, j) \\ 0, & \text { otherwise. }\end{cases}
$$

Then $B=A^{-1}$.

Let $T$ be a nonsingular tree with vertices $1,2, \cdots, n$. Let $T^{-1}$ denote the graph with vertex set $\{1,2, \cdots, n\}$, where vertices $i$ and $j$ are adjacent in $T^{-1}$ if and only if there is an alternating path between $i$ and $j$ in $T$. We call the graph $T^{-1}$ the inverse graph of the nonsingular tree $T$. It is shown in [3] that the graph $T^{-1}$ is connected and bipartite, see [3] for more detail.

Corollary 3.7 (Barik, Neumann and Pati [3, Lemma 2.3]) Let $T$ be a nonsingular tree and $T^{-1}$ be its inverse graph. Then the inverse matrix of the adjacency matrix of $T$ is similar to the adjacency matrix of $T^{-1}$ via a diagonal matrix of \pm 1 . 
Let $P(i, j)$ be an alternating path from vertex $i$ to vertex $j$ of $\vec{T}$ and let $|\vec{P}(i, j)|$ be the number of oriented edges in $P(i, j)$ whose orientation agrees with the routing from $i$ to $j$. Note that if the alternating path $P(i, j)=i_{1} i_{2} i_{3} \cdots i_{2 k}$ (where $i_{1}=i, i_{2 k}=j$ ) then

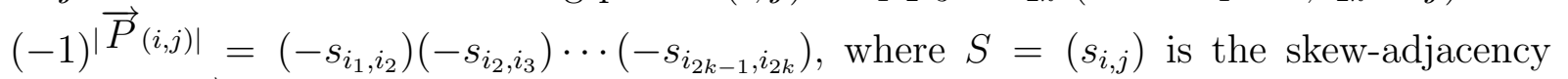
matrix of $\vec{T}$.

Although we are concerned with trees here, it should be mentioned that Proposition 3.6 and Corollary 3.7 have been generalized to bipartite graphs with a unique perfect matching (see [3, Lem. 2.1] and [2, Thm. 5 and Cor.5]. Also, the inverse graph $T^{-1}$ is presented as an example of a graph inverse $G^{+}$defined in [11] (see Thm 3.2 there).

Using a technique similar to that in [3, Lemma 2.1], we obtain the following combinatorial description of the inverse of the skew-adjacency matrix of a tree with a perfect matching.

Lemma 3.8 Let $\vec{T}$ be an orientation of a nonsingular tree $T$ on $n$ vertices and $S$ be its skew-adjacency matrix. Let $R=\left(r_{i, j}\right)$, where

$$
r_{i, j}= \begin{cases}(-1)^{\mid \vec{P}}(i, j) \mid, & \text { if there is an alternating path } P(i, j), \\ 0, & \text { otherwise. }\end{cases}
$$

Then $R=S^{-1}$.

Proof. The $(i, j)$-th entry of $S R$ is given by

$$
(S R)_{i, j}=\sum_{k=1}^{n} s_{i, k} r_{k, j}=\sum_{k \sim i} s_{i, k} r_{k, j}
$$

Thus for each $i=1,2, \ldots, n$,

$$
(S R)_{i, i}=\sum_{k \sim i} s_{i, k} r_{k, i}=s_{i, i^{\prime}}\left(-s_{i^{\prime}, i}\right)=1
$$

as there exists exactly one vertex, say $i^{\prime}$, such that the edge $i^{\prime} i \in \mathcal{M}$.

Now let $i, j$ be two distinct vertices in $T$. Suppose that for each vertex $v$ adjacent to $i$, there is no alternating path from $v$ to $j$, then $r_{v, j}=0$. Thus we have $(S R)_{i, j}=0$. Moreover, $v$ is unique, otherwise there would be a cycle in $T$ containing the vertex $i$.

Assume now that there is a vertex $v \neq i^{\prime}$ adjacent to $i$ such that $P(v, j)=v x_{2} \cdots x_{m-1} j$ is an alternating path from $v$ to $j$. In this case, $P^{\prime}=i^{\prime} i v x_{2} \cdots x_{m-1} j$, that is, $i^{\prime} i P(v, j)$ is also an alternating path from $i^{\prime}$ to $j$. Conversely, if there is an alternating path $P\left(i^{\prime}, j\right)$ from $i^{\prime}$ to $j$, it must have the form $i^{\prime} i v x_{2} \cdots x_{m-1} j$. Thus there must exist a vertex $v \neq i^{\prime}$ adjacent to $i$ such that an alternating path from $v$ to $j$ exists.

We have just seen that the alternating path from $i^{\prime}$ to $j$ is of the form $i^{\prime} i P(v, j)$, where $P(v, j)$ is the alternating path from $v$ to $j$. Hence

$$
(S R)_{i, j}=s_{i, i^{\prime}} r_{i^{\prime}, j}+s_{i, v} r_{v, j}=s_{i, i^{\prime}}\left(-s_{i^{\prime}, i}\right)\left(-s_{i, v}\right) r_{v, j}+s_{i, v} r_{v, j}=0
$$




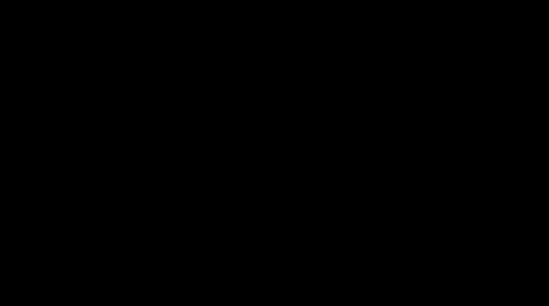

$T$

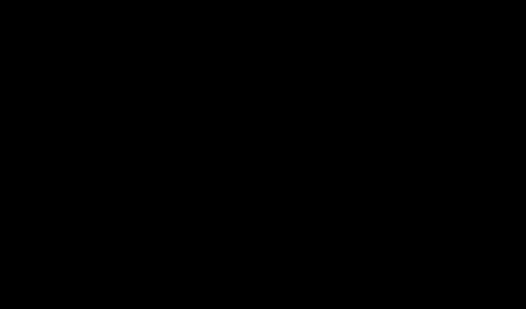

An orientation $\vec{T}$ of $T$

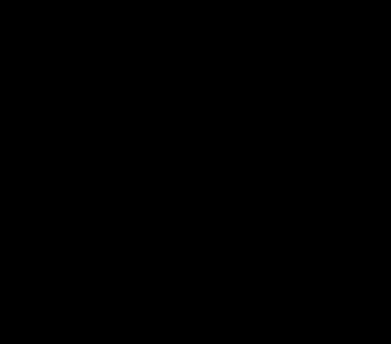

$T^{-1}$

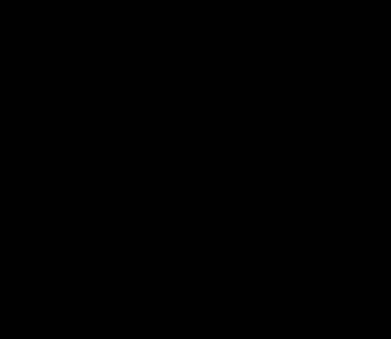

Figure 2: A tree and its inverses

and the proof is done.

From Lemma 3.8, we see that if $S^{-1}$ is the skew-adjacency matrix of an orientation $\vec{T}$ of a tree $T$ with a perfect matchings, then $S^{-1}$ is also a skew symmetric matrix with entries $0,-1$, or 1 . Thus $S^{-1}$ is the skew-adjacency matrix of some oriented graph, we use the notation $\vec{T}^{-1}$ for this oriented graph and call $\vec{T}^{-1}$ the inverse oriented graph of $\vec{T}$. Because of $\left|b_{i j}\right|=\left|r_{i j}\right|$ in Proposition 3.6 and Lemma 3.8, it follows that $\vec{T}^{-1}$ is an orientation of the inverse graph $T^{-1}$ of $T$. See Fig. 2 for an example based on Fig. 1 in [3]. The dotted lines represent the edges in the perfect matching $\mathcal{M}$.

Proposition 3.9 Let $T$ be a tree with a perfect matching and $\vec{T}$ be any orientation of T. Then $S p\left(\vec{T}^{-1}\right)=\mathbf{i} S p\left(T^{-1}\right)$ and hence $\mathcal{E}\left(\vec{T}^{-1}\right)=\mathcal{E}\left(T^{-1}\right)$.

Proof. Let $\lambda_{1}, \lambda_{2}, \ldots, \lambda_{n}$ be all eigenvalues of $T$. Then $\lambda_{1}, \lambda_{2}, \ldots, \lambda_{n}$ are non-zero as $T$ is nonsingular and $S p\left(T^{-1}\right)=\left\{\frac{1}{\lambda_{1}}, \frac{1}{\lambda_{2}}, \cdots, \frac{1}{\lambda_{n}}\right\}$ by Corollary 3.7. As $T$ is a tree, we have $S p(\vec{T})=\left\{\lambda_{1} \mathbf{i}, \lambda_{2} \mathbf{i}, \cdots, \lambda_{n} \mathbf{i}\right\}$ by Theorem 3.4. Thus $S p_{S}\left(\vec{T}^{-1}\right)=$ $\left\{-\frac{1}{\lambda_{1}} \mathbf{i},-\frac{1}{\lambda_{2}} \mathbf{i}, \cdots,-\frac{1}{\lambda_{n}} \mathbf{i}\right\}=\left\{\frac{1}{\lambda_{1}} \mathbf{i}, \frac{1}{\lambda_{2}} \mathbf{i}, \cdots, \frac{1}{\lambda_{n}} \mathbf{i}\right\}$ for the skew-adjacency matrix of $\vec{T}^{-1}$ is the inverse of the skew-adjacency matrix of $\vec{T}$ and the negative of each eigenvalue of $T$ is also an eigenvalue of $T$. Therefore $\operatorname{Sp}\left(\vec{T}^{-1}\right)=\mathbf{i} S p\left(T^{-1}\right)$ and hence $\mathcal{E}\left(\vec{T}^{-1}\right)=\mathcal{E}\left(T^{-1}\right)$. 


\section{Acknowledgment}

The authors would like to express their sincere gratitude to the referee for a very careful reading of the paper and for all his or her insightful comments and valuable suggestions, which make a number of improvements on this paper. The first author was supported by National Natural Science Foundation of China.

\section{References}

[1] C. Adiga, R. Balakrishnan and Wasin So, The skew energy of a digraph, Linear Algebra and its Applications 432 (2010) 1825-1835.

[2] S. Akbari and S. J. Kirkland, On unimodular graphs, Linear Algebra and its Applications 421 (2007) 3-15.

[3] S. Barik, M. Neumann and S. Pati, On nonsingular trees and a reciprocal eigenvalue property, Linear and Mulitilinear Algebra 54 (2006) 453-465.

[4] S. Barik, M. Nath, S. Pati and B. K. Sarma, Unicyclic graphs with the strong reciprocal eigenvalue property, Electronic Journal of Linear Algebra 17 (2008) 139-153.

[5] F. Buckley, L. L. Doty and F. Harary, On graphs with signed inverses, Networks, 18 (1988) 151-157.

[6] R. A. Brualdi and H. J. Ryser, Combinatorial Matrix Theory, Cambridge University Press, Cambridge, 1991.

[7] D. Cvetkovic M. Doob and H. Sachs, Spectra of Graphs, Academic Press, New York, 1980.

[8] C. D. Godsil, Algebraic Combinatorics, Chapman \& Hall, London, 1993.

[9] L. Lovász and M. Plummer, Matching Theory, Ann. of Discrete Math. 29, NorthHolland, New York,1988.

[10] B. Shader and Wasin So, Skew spectra of oriented graphs, The Electronic Journal of Combinatorics 16 (2009), \#N32.

[11] R. M. Tifenbach and S. J. Kirkland, Directed intervals and dual of a graph, Linear Algebra and its Applications 431 (2009) 792-807. 